\title{
Current State and Future Prospects of EEG and fNIRS in Robot-Assisted Gait Rehabilitation: A Brief Review
}

\author{
Alisa Berger ${ }^{1 *}$, Fabian Horst ${ }^{2}$, Sophia Müller ${ }^{1}$, Fabian Steinberg ${ }^{1}$ and \\ Michael Doppelmayr ${ }^{1,3}$ \\ ${ }^{1}$ Department of Sport Psychology, Institute of Sport Science, Johannes Gutenberg-University, Mainz, Germany, \\ ${ }^{2}$ Department of Training and Movement Science, Institute of Sport Science, Johannes Gutenberg-University, Mainz, \\ Germany, ${ }^{3}$ Centre for Cognitive Neuroscience, University of Salzburg, Salzburg, Austria
}

OPEN ACCESS

Edited by:

Zhen Yuan,

University of Macau, China

Reviewed by:

Marco Ferrari,

University of L'Aquila, Italy

Johanna Wagner,

University of California, San Diego,

United States

*Correspondence:

Alisa Berger

alisa.berger@uni-mainz.de

Received: 30 October 2018 Accepted: 13 May 2019

Published: 05 June 2019

Citation:

Berger A, Horst F, Müller S, Steinberg $F$ and Doppelmayr $M$ (2019) Current State and Future

Prospects of EEG and fNIRS in Robot-Assisted Gait Rehabilitation:

A Brief Review.

Front. Hum. Neurosci. 13:172. doi: 10.3389/fnhum.2019.00172
Gait and balance impairments are frequently considered as the most significant concerns among individuals suffering from neurological diseases. Robot-assisted gait training (RAGT) has shown to be a promising neurorehabilitation intervention to improve gait recovery in patients following stroke or brain injury by potentially initiating neuroplastic changes. However, the neurophysiological processes underlying gait recovery through RAGT remain poorly understood. As non-invasive, portable neuroimaging techniques, electroencephalography (EEG) and functional near-infrared spectroscopy (fNIRS) provide new insights regarding the neurophysiological processes occurring during RAGT by measuring different perspectives of brain activity. Due to spatial information about changes in cortical activation patterns and the rapid temporal resolution of bioelectrical changes, more features correlated with brain activation and connectivity can be identified when using fused EEG-fNIRS, thus leading to a detailed understanding of neurophysiological mechanisms underlying motor behavior and impairments due to neurological diseases. Therefore, multi-modal integrations of EEG-fNIRS appear promising for the characterization of neurovascular coupling in brain network dynamics induced by RAGT. In this brief review, we surveyed neuroimaging studies focusing specifically on robotic gait rehabilitation. While previous studies have examined either EEG or fNIRS with respect to RAGT, a multi-modal integration of both approaches is lacking. Based on comparable studies using fused EEG-fNIRS integrations either for guiding non-invasive brain stimulation or as part of brain-machine interface paradigms, the potential of this methodologically combined approach in RAGT is discussed. Future research directions and perspectives for targeted, individualized gait recovery that optimize the outcome and efficiency of RAGT in neurorehabilitation were further derived.

Keywords: multi-modal approach, electroencephalography, functional near-infrared spectroscopy, robotassisted gait training, motor recovery, neurorehabilitation, brain-machine interface, brain stimulation

\section{INTRODUCTION}

According to the World Health Organization, over one billion people are affected by gait and balance impairments due to neurological diseases that impact independent living and quality of life (Stump, 2007; Turner et al., 2013; Calabrò et al., 2016). With respect to maladaptive brain functions in the motor domain (e.g., following stroke), the brain has the remarkable capacity to repair itself to 
a certain extent. Such processes occur through functional and structural changes during motor (re-)learning, which are collectively termed neuroplasticity (Pascual-Leone et al., 2005; Pascual-Leone et al., 2011; Kays et al., 2012; Chen et al., 2015). In recent years, robot-assisted neurorehabilitation has been applied in addition to manual-assisted therapy due to its provision of early, intensive, task-specific, and multi-sensory training, which is thought to be most effective for motor recovery by favoring neuroplastic changes (Turner et al., 2013; Calabrò et al., 2016). Indeed, current literature suggests that patients with neurological diseases improved their walking ability (Calabrò et al., 2016), walking speed (Benito-Penalva et al., 2012; Uçar et al., 2014), leg muscle force (Beer et al., 2008), step length, and gait symmetry (Husemann et al., 2007; Esquenazi et al., 2013) due to robotic rehabilitation. Nevertheless, the science-based evidence for improved gait recovery is mixed due to heterogeneous study designs and methods, coupled with a lack of knowledge regarding the underlying neurophysiological mechanisms of robot-assisted gait training (RAGT) (Swinnen et al., 2010, 2015; Knaepen et al., 2015).

New insights into brain activity during RAGT would allow the improvement of robotic rehabilitation, however, it is a challenge because of movement-related signal artifacts arising during the interaction of robot systems with their users. In contrast to stationary neuroimaging methods such as functional magnet resonance imaging (fMRI) which do not allow the analysis during movements (Gramann et al., 2011), wearable mobile brain imaging (MoBI) systems provide an approach to fully inv estigate how natural, varying behavior is guided by complex neural dynamics (Makeig et al., 2009). EEG and fNIRS are noninvasive, portable, and cost-effective methods representing the MoBI approach by enabling the measurement of brain activity in natural environments.

Electroencephalography records the integrated and synchronized activity of pyramidal neurons in the cerebral cortex, either postsynaptic potentials associated with neural activation [e.g., event-related potentials (ERPs)] or changes and strengths of various oscillation frequencies (i.e., delta, theta, alpha, mu, beta, and gamma) which are expressed as power spectral density or coherence (Knaepen et al., 2015). In addition to an excellent temporal resolution, reasonable spatial accuracy can be ensured by new high-density systems (Robinson et al., 2017) or statistical methods such as independent component analysis (ICA) decompositions by reconstructing the origin of EEG activity (Makeig et al., 2004; Onton et al., 2006; Gramann et al., 2010). Furthermore, artifacts during walking due to head movements, eye movements or neck muscle activity can be detected with ICA (Gwin et al., 2010, 2011; Wagner et al., 2012; Snyder et al., 2015) or artifact removal methods including moving average and wavelet-based techniques (Kline et al., 2015), thus, resulting in improved signal-to-noise ratio. Studies investigating brain oscillations during walking (Petersen et al., 2012; Severens et al., 2012; Solis Escalante et al., 2012; Wagner et al., 2012, 2016; Sanctis et al., 2014; Seeber et al., 2014; Bulea et al., 2015) showed sustained $\mathrm{mu}$ and beta desynchronization within sensorimotor cortex (SMC; Severens et al., 2012; Wagner et al., 2012, 2014; Seeber et al., 2014; Bulea et al., 2015) demonstrating that motor cortex and corticospinal tract contribute directly to the muscle activity in locomotion (Petersen et al., 2012).

In contrast to EEG, fNIRS relies on the principle of neurovascular coupling measuring changes in regional cerebral blood flow, oxygenated hemoglobin (Hboxy), and deoxygenated hemoglobin (Hbdeoxy) induced by neuronal activation, which is analogous to the blood-oxygenation-level-dependent responses measured by fMRI (Ferrari et al., 2004). Both provide information regarding the spatial location of the recorded activity, whereas the temporal resolution is limited due to the intrinsically slow processes of hemodynamic changes. Compared to fMRI, fNIRS is - although with lower resolution (Koch et al., 2008) affordable and easily implementable in a portable system, thus facilitating a wider range of applications. Recently, wearable and wireless systems were developed which allow the topographical representation of hemodynamic responses over the cortical surface due to high-density multi-distance channels (Shin et al., 2017). Furthermore, they are lightweight and robust to motion artifacts due to the absence of fiber-optic bundles (Pinti et al., 2018). Despite advantages and developments, the challenge remains to distinguish physiological changes through brain activity from noise and artifacts. Various methods have been proposed recently to correct for motion artifacts, including principle component analysis, spline interpolation, Kalman filtering, wavelet filtering and correlation-based signal improvement, with the result that wavelet filtering seems be the most promising and powerful technique for motion artifact correction in fNIRS data (Brigadoi et al., 2014). Furthermore, new short channels systems enable the detection of scalphemodynamics contaminating the fNIRS signal which can be removed from cerebral hemodynamics of long-channels using general linear model to estimate brain activity (Sato et al., 2016). In studies investigating brain activity in terms of Hboxy/Hbdeoxy concentration changes during walking, healthy people had significantly increased brain activity in SMC, premotor cortex (PMC), supplementary motor area (SMA) as well as prefrontal cortex (PFC) (Miyai et al., 2001; Hamacher et al., 2015). In comparison, patients with neurological diseases and elderly people showed increased PFC activity associated with low gait capacity (Harada et al., 2009; Stuart et al., 2018). A standardized method to identify and reduce motion as well as physiological artifacts (e.g., heart rate, mayer waves, respiration) during walking is missing so far (Vitorio et al., 2017).

Based on the independence between electrical and optical measurements as well as the assets and drawbacks of each neuroimaging technique, the multi-modal integration of EEGfNIRS can partially overcome the limitations encountered by each individual modality (Merzagora et al., 2009; Muthalib et al., 2013), which may help to more accurately characterize the functional activity of neural networks involved in robotic rehabilitation. However, although RAGT is the most frequent robot-assisted intervention for neurological injuries, little is known regarding the neural correlates of gait recovery in RAGT (Kim et al., 2016). In order to determine optimal training parameters for individualized gait therapy protocols, it is essential to understand how robotic devices interact with their users, and thus, how both locomotor control and gait recovery is 
characterized by brain signals (Solis Escalante et al., 2012; Youssofzadeh et al., 2014, 2016).

Therefore, we (1) surveyed neuroimaging studies focusing on RAGT (summarized in Table 1) with the aim to contribute to the understanding of neurovascular coupling phenomena and (2) inferred the potential of multi-modal integration of EEG-fNIRS in robotic gait rehabilitation (see section Future Prospects for Fused EEG-fNIRS in RAGT).

\section{REVIEW CRITERIA}

The present mini review article focuses on studies addressing EEG or fNIRS during RAGT, thus investigating oscillatory and hemodynamic activity during robot-assisted gait were the decisive criterions. We followed the Preferred Reporting Items for Systematic Reviews statements (Moher et al., 2009) to identify and screen the articles. Figure 1 presents the search strategy as well as the selection criteria in detail. A total of 14 articles matched the inclusion criteria. All relevant data extracted and summarized from the screening process were shown in Table 1.

\section{NEURAL CORRELATES OF RAGT}

Based on 113 studies identified through database searching, 27 studies were deemed eligible for full-text assessment based on abstract review with 14 studies meeting our final inclusion criteria (see Figure 1). Twelve studies used EEG, two studies measured with fNIRS (see Table 1).

In EEG studies, the Lokomat (Hocoma, Switzerland) was used most frequently (Lapitskaya et al., 2011; Wagner et al., 2012; Seeber et al., 2013, 2014, 2015; Knaepen et al., 2015) whereas the active leg exoskeleton (ALEX; Youssofzadeh et al., 2014, 2016) was used in two studies and the gait assistance robot (GAR; Nakanishi et al., 2014), H2 exoskeleton (VillaParra et al., 2015; Contreras-Vidal et al., 2018) or the wearable Ekso $^{\mathrm{TM}}$ (Calabrò et al., 2018) in one study only. Changes in electrophysiological activity due to RAGT were analyzed by investigating either event-related potentials, power-spectral densities as well as coherence. Examinations were carried out either with healthy volunteers or in three studies with neurologically ill patients.

In healthy subjects, Wagner et al. (2012) investigated spectral patterns during active and passive robot-assisted walking and showed significantly suppressed mu $(8-12 \mathrm{~Hz})$ and beta (18$21 \mathrm{~Hz}$ ) rhythms in central midline areas during active walking that also depend on gait cycle phases. These results have been underpinned by suppressed $\mathrm{mu}(10-12 \mathrm{~Hz})$ and beta (18$30 \mathrm{~Hz}$ ) oscillations (Seeber et al., 2013) as well as increased gamma amplitudes $(24-40 \mathrm{~Hz})$ during robot-assisted walking compared to standing (Seeber et al., 2015) or reduced power in alpha and beta bands during active participation due to

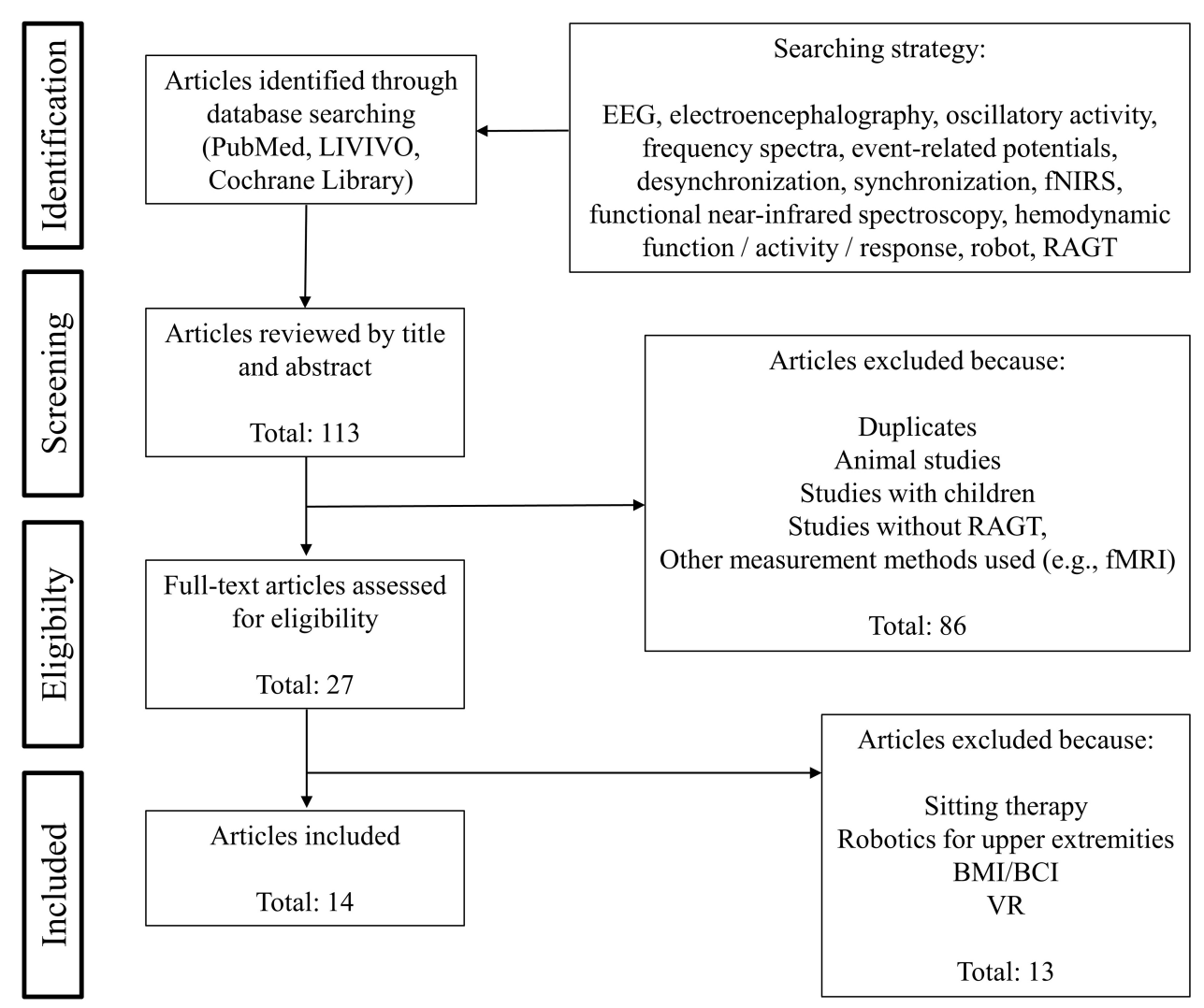

FIGURE 1 | Flowchart of the article selection process. 
TABLE 1 | Overview of studies investigating the neurophysiological mechanisms underlying RAGT using EEG and fNIRS.

\begin{tabular}{|c|c|c|c|c|c|c|c|c|}
\hline Author(s) & Title & Robotic system & Method & EEG/fNIRS device & Parameter & Sample & Outcome & Conclusion \\
\hline $\begin{array}{l}\text { Calabrò et al., } \\
2018\end{array}$ & $\begin{array}{l}\text { Shaping } \\
\text { neuroplasticity by } \\
\text { using powered } \\
\text { exoskeletons in } \\
\text { patients with } \\
\text { stroke: a } \\
\text { randomized clinical } \\
\text { trial }\end{array}$ & $\mathrm{Ekso}^{\mathrm{TM}}$ & $\begin{array}{l}\text { Prospective, } \\
\text { pre-post, } \\
\text { randomized clinical } \\
\text { trial; 1st group: } \\
\text { EGT and OGT; 2nd } \\
\text { group: OGT; Study } \\
\text { design: } 45 \text { min per } \\
\text { session, five times a } \\
\text { week over } 8 \text { weeks }\end{array}$ & $\begin{array}{l}\text { EEG Brain Quick } \\
\text { SystemPLUS } \\
\text { (Micro-med; } \\
\text { Mogliano Veneto, } \\
\text { Italy); } 21 \text { electrodes } \\
\text { Sampling rate: } \\
512 \text { Hz; Electrode } \\
\text { placement: } 10-20 \\
\text { system; Additional: } \\
\text { sEMG \& TMS }\end{array}$ & $\begin{array}{l}\text { EEG: FPEC EMG: } \\
\text { gait performance } \\
\text { based on the } 10 \mathrm{~m} \\
\text { walking test, gait } \\
\text { cycle, muscle } \\
\text { activation muscles; } \\
\text { TMS: CSE and SMI } \\
\text { from both M1 }\end{array}$ & $\begin{array}{l}\text { Stroke patients; } \\
\text { Hemiparesis } \\
\text { caused by stroke in } \\
\text { a chronic phase; } \\
\text { Number of } \\
\text { participants: } 40 \\
\text { patients, } 20 \text { each in } \\
\text { a group; Age: } \geq 55 \\
\text { years }\end{array}$ & $\begin{array}{l}\text { FPEC: significant } \\
\text { strengthening of } \\
\text { EGT group; CSE: } \\
\text { significant } \\
\text { improvement in } \\
\text { EGT group on the } \\
\text { affected side; SMl: } \\
\text { significant } \\
\text { improvement in } \\
\text { EGT group on } \\
\text { affected side; } 10 \text { m } \\
\text { walk test: } \\
\text { significant } \\
\text { improvement in } \\
\text { EGT group; } \\
\text { General walking } \\
\text { quality: significant } \\
\text { improvement; Hip } \\
\text { and knee muscle } \\
\text { activation: } \\
\text { significant } \\
\text { improvement }\end{array}$ & $\begin{array}{l}\text { EGT seems, in addition } \\
\text { to OGT, also promising } \\
\text { in gait rehabilitation for } \\
\text { patients after a stroke. } \\
\text { The study suggests } \\
\text { that Ekso could be } \\
\text { useful to promote the } \\
\text { mobility of people with } \\
\text { stroke, thanks to the } \\
\text { mechanisms of brain } \\
\text { plasticity and } \\
\text { remodulation of } \\
\text { connectivity that are } \\
\text { specifically carried } \\
\text { along by the robotic } \\
\text { system compared to } \\
\text { conventional OGT. }\end{array}$ \\
\hline $\begin{array}{l}\text { Contreras-Vidal } \\
\text { et al., } 2018\end{array}$ & $\begin{array}{l}\text { Neural Decoding of } \\
\text { Robot-Assisted } \\
\text { Gait during } \\
\text { Rehabilitation after } \\
\text { Stroke }\end{array}$ & H2 Exoskelett & $\begin{array}{l}\text { Study design: } 12 \\
\text { training sessions, } 4 \\
\text { weeks Meanwhile, } \\
\text { no additional } \\
\text { therapy; Walking } \\
\text { speed: preset and } \\
\text { comfortable for the } \\
\text { test persons, could } \\
\text { be adjusted while } \\
\text { walking }\end{array}$ & $\begin{array}{l}\text { EEG BrainAmpDC, } \\
\text { Brain Products, } \\
\text { Germany; } 64 \\
\text { electrodes } \\
\text { Sampling rate: } \\
1000 \mathrm{~Hz}\end{array}$ & $\begin{array}{l}\text { Changes in power } \\
\text { spectra for the } \\
0.1-3 \mathrm{~Hz} \text { (delta) } \\
\text { band; Additional: } \\
\text { walking speed, } \\
\text { walking distance }\end{array}$ & $\begin{array}{l}\text { Stroke patients } \\
\text { Chronic } \\
\text { hemiparesis after a } \\
\text { stroke Number of } \\
\text { test persons: } 5 \text {; } \\
\text { Exclusion: } 1\end{array}$ & $\begin{array}{l}\text { Suppression of the } \\
\text { delta frequency } \\
\text { when walking in the } \\
\text { occipital scalp area. } \\
\text { Increase in delta } \\
\text { frequency } \\
\text { suppression in the } \\
\text { frontal and central } \\
\text { scalp regions. } \\
\text { Improvement of } \\
\text { walking distance } \\
\text { and walking speed, } \\
\text { which correlated } \\
\text { with increased } \\
\text { accuracy of offline } \\
\text { decoding. }\end{array}$ & $\begin{array}{l}\text { Proof of the feasibility of } \\
\text { neuronal decoding of } \\
\text { gait kinematics from the } \\
\text { EEG during RAGT in } \\
\text { chronic stroke patients. } \\
\text { Since motor intention } \\
\text { recognition from EEG } \\
\text { signals is synchronized } \\
\text { with motion feedback } \\
\text { generated by } \\
\text { exoskeleton-assisted } \\
\text { movements of the } \\
\text { lower extremities, the } \\
\text { BMI-H2 system can } \\
\text { promote brain } \\
\text { reorganization through } \\
\text { motor learning, } \\
\text { presumably due to } \\
\text { activity-dependent } \\
\text { brain plasticity. First } \\
\text { step in the } \\
\text { development of a } \\
\text { brain-machine interface } \\
\text { to control driven } \\
\text { exoskeletons. }\end{array}$ \\
\hline
\end{tabular}


TABLE 1 | Continued

\begin{tabular}{|c|c|c|c|c|c|c|c|c|}
\hline Author(s) & Title & Robotic system & Method & EEG/fNIRS device & Parameter & Sample & Outcome & Conclusion \\
\hline $\begin{array}{l}\text { Knaepen et al., } \\
2015\end{array}$ & $\begin{array}{l}\text { Human-Robot } \\
\text { Interaction: Does } \\
\text { Robotic Guidance } \\
\text { Force Affect } \\
\text { Gait-Related Brain } \\
\text { Dynamics during } \\
\text { Robot-Assisted } \\
\text { Treadmill Walking? }\end{array}$ & Lokomat & $\begin{array}{l}\text { Stratified randomization } \\
4 \text { gait conditions of } \\
5 \text { min each: } 1 \mathrm{st} \\
\text { condition at the } \\
\text { beginning without } \\
\text { Lokomat. Condition } \\
2-4 \text { with Lokomat with } \\
3 \text { levels of managers. } \\
\text { Speed: } 2 \text { km/h; } \\
\text { Conditions: Unassisted } \\
\text { treadmill walking as well } \\
\text { as during } \\
\text { robot-assisted treadmill } \\
\text { walking GF: } 30,60 \text {, } \\
\text { and } 100 \% \text { BWS: } 0\end{array}$ & $\begin{array}{l}\text { EEG BrainAmp DC } \\
\text { \& BrainVision } \\
\text { Recorder, Brain } \\
\text { Products GmbH, } \\
\text { Germany; } 32 \\
\text { electrodes } \\
\text { Sampling rate: } \\
1000 \mathrm{~Hz} \text { Band } \\
\text { pass: } 0.5-100 \mathrm{~Hz} \text {; } \\
\text { Electrode } \\
\text { placement: } 10-20 \\
\text { system }\end{array}$ & $\begin{array}{l}\text { Examination of } \\
\text { ERSPs and PSDs } \\
\text { during RATW at } 30 \text {, } \\
60 \text {, and } 100 \% \text { GF }\end{array}$ & $\begin{array}{l}\text { No health } \\
\text { impairments; } \\
\text { Number of test } \\
\text { persons: 11; } \\
\text { Exclusion: 7; Male: } \\
\text { 3; Female: 9; Age: } \\
28.2 \pm 4.0 \text { years; } \\
\text { Weight: } \\
64,7 \pm 7,7 \mathrm{Kg}\end{array}$ & $\begin{array}{l}\text { Gait-related spectral } \\
\text { modulations in the mu-, } \\
\text { beta- and lower } \\
\text { gamma bands above } \\
\text { the SMC, related to } \\
\text { certain phases of the } \\
\text { gait cycle. Mu and beta } \\
\text { rhythms were } \\
\text { suppressed in the right } \\
\text { primary sensory cortex } \\
\text { during treadmill walking } \\
\text { compared to } 100 \% \\
\text { robot-assisted treadmill } \\
\text { training, indicating } \\
\text { significantly greater } \\
\text { involvement of the } \\
\text { sensorimotor area } \\
\text { during treadmill walking } \\
\text { compared to } \\
\text { robot-assisted treadmill } \\
\text { walking. Minor } \\
\text { differences in the } \\
\text { spectral performance of } \\
\text { mu, beta and lower } \\
\text { gamma bands between } \\
\text { robotic treadmill } \\
\text { walking with different } \\
\text { guidance strengths. }\end{array}$ & $\begin{array}{l}\text { High leadership } \\
\text { strength and thus } \\
\text { less active } \\
\text { participation in the } \\
\text { movement should } \\
\text { be avoided during } \\
\text { robot-supported } \\
\text { treadmill training. } \\
\text { This will optimize } \\
\text { the participation of } \\
\text { the sensorimotor } \\
\text { cortex, which is } \\
\text { known to be } \\
\text { essential for motor } \\
\text { learning. }\end{array}$ \\
\hline
\end{tabular}


TABLE 1 | Continued

\begin{tabular}{|c|c|c|c|c|c|c|c|c|}
\hline Author(s) & Title & Robotic system & Method & EEG/fNIRS device & Parameter & Sample & Outcome & Conclusion \\
\hline $\begin{array}{l}\text { Lapitskaya } \\
\text { et al., } 2011\end{array}$ & $\begin{array}{l}\text { Robotic gait training in } \\
\text { patients with impaired } \\
\text { consciousness due to } \\
\text { severe traumatic brain injury }\end{array}$ & Lokomat & $\begin{array}{l}\text { Prospective, } \\
\text { controlled, } \\
\text { non-randomized } \\
\text { study. Single } \\
\text { training session: } \\
\text { Speed: } \\
1.5 \pm 0.1 \mathrm{~km} / \mathrm{h} \\
\text { (patients), } \\
1.61 \pm 0.08 \mathrm{~km} / \mathrm{h} \\
\text { (healthy subjects); } \\
\text { Training time: } \\
17.1 \pm 1.3 \text { min } \\
\text { (patients), } \\
17.15 \pm 0.11 \text { min } \\
\text { (healthy volunteers); } \\
\text { Walking distance: } \\
427.3 \pm 38.6 \mathrm{~m} \\
\text { (patients), } \\
451.36 \pm 15.02 \mathrm{~m} \\
\text { (healthy volunteers); } \\
\text { conditions: } \\
\text { Recovery phases } \\
\text { and RAGT; GF: } \\
100 \% \text {; EEG } \\
\text { recording in sitting } \\
\text { position }\end{array}$ & $\begin{array}{l}\text { EEG Nervous } \\
\text { system } \\
\text { (Taugagreining hf, } \\
\text { Reykjavik, Island) } \\
19 \text { electrodes } \\
\text { placement: Fz, Cz } \\
\text { and Pz; ECl } \\
\text { Electro-Cap } \\
\text { SystemTM, } \\
\text { International, Inc., } \\
\text { Eaton, OH, } \\
\text { United States; SEP } \\
\text { measurement: } \\
\text { 'VikingQuest' } \\
\text { (Viasys Healthcare, } \\
\text { San Diego, CA, } \\
\text { United States) }\end{array}$ & $\begin{array}{l}\text { Sensory nerve } \\
\text { tracts were } \\
\text { evaluated with the } \\
\text { help of sensory } \\
\text { ERPs. Global DAR } \\
\text { and the latency of } \\
\text { the P300 } \\
\text { component of the } \\
\text { event-related } \\
\text { potentials before } \\
\text { and after a training } \\
\text { session. }\end{array}$ & $\begin{array}{l}\text { Patients with TBI } \\
\text { disturbances of } \\
\text { consciousness; } \\
\text { Sample size: } 12 ; \\
\text { Male: } 9 \text {; Female: } 3 \text {; } \\
\text { Age: } 40.8 \pm 18.2 \\
\text { years; Control } \\
\text { group: } 14 \text { healthy } \\
\text { male subjects; Age: } \\
47.3 \pm 14.5 \text { years }\end{array}$ & $\begin{array}{l}\text { Basic } \\
\text { measurement: } \\
\text { Impaired SEPs in } \\
\text { most patients and a } \\
\text { significantly larger } \\
\text { DAR in patients } \\
\text { compared to } \\
\text { healthy ones; After } \\
\text { RAGT: Reduction of } \\
\text { DAR in healthy } \\
\text { subjects, but not in } \\
\text { patients. No } \\
\text { changes in P300 } \\
\text { latency after } \\
\text { training in patients } \\
\text { or healthy subjects. }\end{array}$ & $\begin{array}{l}\text { The study showed } \\
\text { that robotic gait } \\
\text { training induces } \\
\text { measurable } \\
\text { changes in the EEG } \\
\text { performance } \\
\text { spectrum in healthy } \\
\text { individuals, while no } \\
\text { changes were } \\
\text { observed in } \\
\text { patients with severe } \\
\text { TBl. The absence } \\
\text { of changes in the } \\
\text { EEG power } \\
\text { spectrum after } \\
\text { RAGT in the patient } \\
\text { may be an indicator } \\
\text { of the severity of } \\
\text { the injury. }\end{array}$ \\
\hline $\begin{array}{l}\text { Nakanishi et al., } \\
2014\end{array}$ & $\begin{array}{l}\text { Rapid changes in arousal } \\
\text { states of healthy volunteers } \\
\text { during robot-assisted gait } \\
\text { training: a quantitative } \\
\text { time-series } \\
\text { electroencephalography } \\
\text { study }\end{array}$ & GAR & $\begin{array}{l}\text { Conditions: } \\
\text { Standing versus } \\
\text { passive RAGT } \\
\text { Standing: } 30 \text { s with } \\
\text { eyes closed and } 30 \\
\text { s with eyes open; } \\
\text { RAGT: } 6 \text { min. at } 3 \\
\text { conditions: (1) sinus } \\
\text { wave noise } \\
\text { stimulation, (2) } \\
\text { verbal noise } \\
\text { stimulation, (3) no } \\
\text { noise stimulation; } \\
\text { Speed: } 0.11 \mathrm{~m} / \mathrm{s}\end{array}$ & $\begin{array}{l}\text { EEG Polymate ॥ } \\
\text { AP216, TEAC, } \\
\text { Tokyo, Japan } \\
\text { Sampling rate: } \\
1000 \mathrm{~Hz} \text {; Electrode } \\
\text { placement: 10-20 } \\
\text { system }\end{array}$ & $\begin{array}{l}\text { The PSD of the } \\
\text { theta, alpha- } 1 \text { and } \\
\text { alpha-2 bands } \\
\text { were calculated as } \\
\text { indicators of } \\
\text { objective } \\
\text { drowsiness. }\end{array}$ & $\begin{array}{l}\text { No health } \\
\text { impairments; } \\
\text { Sample size: 12; All } \\
\text { male Age: } \\
39.3 \pm 1.8 \text { years; } \\
\text { Weight: } \\
64.9 \pm 2.3 \mathrm{~kg} ; \\
\text { Body height: } \\
168.4 \pm 0.8 \mathrm{~cm}\end{array}$ & $\begin{array}{l}\text { Increase power } \\
\text { density in theta } \\
(4.0-7.9 \mathrm{~Hz}) \text { and } \\
\text { alpha bands (ERS). }\end{array}$ & $\begin{array}{l}\text { EEG-measured } \\
\text { excitation values } \\
\text { during RAGT } \\
\text { decreased within a } \\
\text { short time but can } \\
\text { be restored and } \\
\text { maintained by } \\
\text { intermittent warning } \\
\text { tone stimulation. }\end{array}$ \\
\hline
\end{tabular}


TABLE 1 | Continued

\begin{tabular}{|c|c|c|c|c|c|c|c|c|}
\hline Author(s) & Title & Robotic system & Method & EEG/fNIRS device & Parameter & Sample & Outcome & Conclusion \\
\hline $\begin{array}{l}\text { Seeber et al., } \\
2013\end{array}$ & $\begin{array}{l}\text { Spatial-Spectral } \\
\text { Identification of } \mu \text { and } \beta \\
\text { EEG Rhythm Sources } \\
\text { During Robot-Assisted } \\
\text { Walking }\end{array}$ & Lokomat & $\begin{array}{l}\text { Conditions: } 3 \text { runs } \\
\text { standing upright, } \\
3 \text { min each; } 4 \text { runs } \\
\text { active walking, } \\
6 \text { min each }\end{array}$ & $\begin{array}{l}\text { EEG } 120 \\
\text { electrodes } \\
\text { Sampling rate: } \\
2.5 \mathrm{kHz} \text {; High pass: } \\
0.1 \mathrm{~Hz} \text { : Low pass: } \\
1000 \mathrm{~Hz} \text {; Electrode } \\
\text { placement: } 10-20 \\
\text { system }\end{array}$ & $\begin{array}{l}\text { Power spectra } \\
\text { (ERD); Functional } \\
\text { brain topography }\end{array}$ & $\begin{array}{l}\text { No health } \\
\text { impairments; } \\
\text { Sample size: 8; } \\
\text { Male: } 5 \text {; Female: 3; } \\
\text { Age: } 26.3 \pm 3.5 \\
\text { years }\end{array}$ & $\begin{array}{l}\text { Individual mu and } \\
\text { beta ERD activities } \\
\text { in SMC. The } \\
\text { beta-ERD is more } \\
\text { focal and } \\
\text { consistent among } \\
\text { the test persons in } \\
\text { the foot area than } \\
\text { the mu-ERD. }\end{array}$ & $\begin{array}{l}\text { A method capable } \\
\text { of considering } \\
\text { individual slight } \\
\text { differences in the } \\
\text { rhythms mu and } \\
\text { beta and locating } \\
\text { the ERD activity of } \\
\text { these rhythms at } \\
\text { the cortical level. } \\
\text { Maximum } \\
\text { frequencies of ERD } \\
\text { were successfully } \\
\text { identified for each } \\
\text { subject in the } \\
\text { frequency range } \\
\text { mu and beta. The } \\
\text { resulting spectral } \\
\text { peaks lead to mu } \\
\text { and beta } \\
\text { topographies for } \\
\text { these frequencies. }\end{array}$ \\
\hline $\begin{array}{l}\text { Seeber et al., } \\
2014\end{array}$ & $\begin{array}{l}\text { EEG beta suppression } \\
\text { and low gamma } \\
\text { modulation are different } \\
\text { elements of human } \\
\text { upright walking }\end{array}$ & Lokomat & $\begin{array}{l}\text { Conditions: } 4 \text { runs } \\
\text { active walking } \\
6 \text { min each, } 3 \text { runs } \\
\text { upright standing } \\
3 \text { min each. Speed: } \\
1.8 \mathrm{~km} / \mathrm{h} \text { - } \\
2.2 \mathrm{~km} / \mathrm{h} \text { (constant, } \\
\text { adapted to the test } \\
\text { persons) BWS: } \\
<30 \% \text { GF: } 100 \%\end{array}$ & $\begin{array}{l}\text { EEG BrainAmp, } \\
\text { Brain-products, } \\
\text { Germany; } 4 \times \\
\text { 32-channel } \\
\text { amplifiers } \\
\text { combined to } 120 \\
\text { channels; Sampling } \\
\text { rate: } 2.5 \mathrm{kHz} \text { Band } \\
\text { pass: } 0.1-1000 \mathrm{~Hz} \text {; } \\
\text { Electrode } \\
\text { placement: } 10-20 \\
\text { system (EasyCap, } \\
\text { Germany) }\end{array}$ & $\begin{array}{l}\text { Amplitude } \\
\text { Modulation \& } \\
\text { Power Spectra } \\
\text { (ERD) }\end{array}$ & $\begin{array}{l}\text { No health } \\
\text { impairments; } \\
\text { Sample size: 10; } \\
\text { Male: 5; Female: 5; } \\
\text { Age: } 25.6 \pm 3.5 \\
\text { years }\end{array}$ & $\begin{array}{l}\text { During active } \\
\text { walking, mu } \\
\text { (10-12 } \mathrm{Hz} \text { ) and } \\
\text { beta (18-30 Hz) } \\
\text { oscillations were } \\
\text { suppressed (ERD) } \\
\text { compared to } \\
\text { standing upright. } \\
\text { Significant beta } \\
\text { ERD was visible in } \\
\text { 9/10 subjects in } \\
\text { central } \\
\text { sensomotoric } \\
\text { areas. Low gamma } \\
\text { (24-40 Hz) } \\
\text { amplitude were } \\
\text { modulated in } \\
\text { relation to the gait } \\
\text { cycle phase. }\end{array}$ & $\begin{array}{l}\text { Persistent mu and } \\
\text { beta ERD reflect a } \\
\text { motion-related } \\
\text { state change in } \\
\text { cortical excitability, } \\
\text { while gait-phase } \\
\text { modulations in the } \\
\text { lower gamma } \\
\text { represent the } \\
\text { motion sequence } \\
\text { time during } \\
\text { walking. }\end{array}$ \\
\hline
\end{tabular}


TABLE 1 | Continued

\begin{tabular}{|c|c|c|c|c|c|c|c|c|}
\hline Author(s) & Title & Robotic system & Method & EEG/fNIRS device & Parameter & Sample & Outcome & Conclusion \\
\hline $\begin{array}{l}\text { Seeber et al., } \\
2015\end{array}$ & $\begin{array}{l}\text { High and low gamma } \\
\text { EEG oscillations in } \\
\text { central sensorimotor } \\
\text { areas are conversely } \\
\text { modulated during the } \\
\text { human gait cycle }\end{array}$ & Lokomat & $\begin{array}{l}\text { Randomized } 8 \text { runs } \\
\text { RAGT } 6 \text { min each } \\
\text { ( } 4 \text { with active } \\
\text { walking and } 4 \text { with } \\
\text { passive walking), } 3 \\
\text { runs standing } \\
\text { upright. } 3 \text { min rest } \\
\text { between the runs. } \\
\text { Speed: } 1.8 \mathrm{~km} / \mathrm{h} \text { - } \\
2.2 \mathrm{~km} / \mathrm{h} \text {; GF: } \\
100 \% \text { BWS: } \\
<30 \%\end{array}$ & $\begin{array}{l}\text { BrainAmp amplifier, } \\
\text { Brain-products, } \\
\text { Germany; } 4 \times 32 \\
\text { channel amplifiers } \\
\text { combined to } 120 \\
\text { channels. Sampling } \\
\text { rate: } 2.5 \mathrm{kHz} \text {; Band } \\
\text { pass: } 0.1-1000 \mathrm{~Hz} \text {; } \\
\text { Electrode } \\
\text { placement: } 10-20 \\
\text { system (EasyCap, } \\
\text { Germany); } \\
\text { Additional: EMG }\end{array}$ & $\begin{array}{l}\text { Temporal dynamics } \\
\text { of EEG oscillations } \\
\text { in the source space } \\
\text { by using } \\
\text { time-frequency } \\
\text { decomposition; } \\
\text { Amplitude } \\
\text { differences } \\
\text { between walking } \\
\text { and standing in mu } \\
\text { (10-12 Hz), beta } \\
\text { (18-30 Hz, gamma } \\
\text { (60-80 Hz) and low } \\
\text { gamma } 24-40 \mathrm{~Hz}) \text {, } \\
\text { high gamma } \\
(70-90 \mathrm{~Hz})\end{array}$ & $\begin{array}{l}\text { No health } \\
\text { impairments; } \\
\text { Sample size: } 10 \text {; } \\
\text { Male: } 5 \text {; Female: } 5 \text {; } \\
\text { Age: } 25.6 \pm 3.5 \\
\text { years right-handed }\end{array}$ & $\begin{array}{l}\text { Increased gamma } \\
(60-80 \mathrm{~Hz}) \\
\text { amplitudes in } \\
\text { central SMC and } \\
\text { modulation of high } \\
\text { gamma during } \\
\text { walking compared } \\
\text { to standing; High } \\
\text { gamma and low } \\
\text { gamma amplitudes } \\
\text { are both modulated } \\
\text { in relation to the } \\
\text { gait cycle, but } \\
\text { conversely to each } \\
\text { other }\end{array}$ & $\begin{array}{l}\text { Altered synchrony } \\
\text { state during } \\
\text { walking compared } \\
\text { to standing due to } \\
\text { static increase of } \\
\text { gamma amplitudes; } \\
\text { Dynamic, amplitude } \\
\text { modulations at } \\
70-90 \text { Hz during } \\
\text { gait cycle may } \\
\text { reflect gait phase } \\
\text { dependent } \\
\text { interactions in } \\
\text { locomotor network; } \\
\text { Distinction of high } \\
\text { and low gamma } \\
\text { amplitudes in } \\
\text { walking } \\
\text { experiments due to } \\
\text { its negative } \\
\text { correlation }\end{array}$ \\
\hline $\begin{array}{l}\text { Villa-Parra } \\
\text { et al., } 2015\end{array}$ & $\begin{array}{l}\text { Toward a robotic knee } \\
\text { exoskeleton control } \\
\text { based on human } \\
\text { motion intention } \\
\text { through EEG and } \\
\text { sEMGsignals }\end{array}$ & $\begin{array}{l}\text { H2 Exoskelett } \\
\text { Additional: UFES's } \\
\text { Smart Walker }\end{array}$ & $\begin{array}{l}\text { Conditions: Sit } \\
\text { down/stand up; } \\
\text { Knee } \\
\text { flexion/extension; } \\
60 \text { trials per } 10 \mathrm{~s}, \\
3 \text { min rest between } \\
\text { conditions }\end{array}$ & $\begin{array}{l}\text { EEG BrainNet BNT } \\
36 \text { electrodes; } \\
\text { Additional sEMG }\end{array}$ & $\begin{array}{l}\text { EEG: HMI analysis } \\
\text { using: ERD/ERS \& } \\
\text { ERP (slow cortical } \\
\text { potentials); sEMG: } \\
\text { myoelectric pattern } \\
\text { classification with } \\
\text { regard to the lower } \\
\text { limb }\end{array}$ & $\begin{array}{l}\text { No health } \\
\text { impairments; } \\
\text { Sample size: 4; All } \\
\text { male }\end{array}$ & $\begin{array}{l}\text { Highest beta ERD } \\
\text { in the range from } \\
20 \text { to } 24 \mathrm{~Hz} \text {; } \\
\text { Highest beta ERS } \\
\text { in the range from } \\
16 \text { to } 22 \mathrm{~Hz}\end{array}$ & $\begin{array}{l}\text { Combination of } \\
\text { EEG/sEMG signals } \\
\text { can be used to } \\
\text { define a control } \\
\text { strategy for the } \\
\text { robot system }\end{array}$ \\
\hline
\end{tabular}


TABLE 1 | Continued

\begin{tabular}{|c|c|c|c|c|c|c|c|c|}
\hline Author(s) & Title & Robotic system & Method & EEG/fNIRS device & Parameter & Sample & Outcome & Conclusion \\
\hline $\begin{array}{l}\text { Wagner et al., } \\
2012\end{array}$ & $\begin{array}{l}\text { Level of participation in } \\
\text { robotic-assisted } \\
\text { treadmill walking } \\
\text { modulates midline } \\
\text { sensorimotor EEG } \\
\text { rhythms in able-bodied } \\
\text { subjects }\end{array}$ & Lokomat & $\begin{array}{l}\text { Randomized } 8 \text { runs } \\
\text { RAGT } 6 \text { min each } \\
\text { ( } 4 \text { with active } \\
\text { walking and } 4 \text { with } \\
\text { passive walking), } 3 \\
\text { runs standing } \\
\text { upright. } 3 \text { min rest } \\
\text { between the runs. } \\
\text { Speed: } 1.8 \mathrm{~km} / \mathrm{h} \text { - } \\
2.2 \mathrm{~km} / \mathrm{h} \text {; GF: } \\
100 \% \text {; BWS: } \\
<30 \%\end{array}$ & $\begin{array}{l}\text { EEG BrainAmp DC } \\
\text { and MR plus } \\
\text { amplifier, } \\
\text { Brain-products, } \\
\text { Germany; } 4 \times 32 \\
\text { channel amplifiers } \\
\text { combined to } 120 \\
\text { channels. Sampling } \\
\text { rate: } 2.5 \mathrm{kHz} \text {; Band } \\
\text { pass: } 0.1-1000 \mathrm{~Hz} \text {; } \\
\text { Electrode } \\
\text { placement: } 10-20 \\
\text { system (EasyCap, } \\
\text { Germany); } \\
\text { Additional: EMG }\end{array}$ & $\begin{array}{l}\text { Power spectra } \\
\text { relating to the } \\
\text { active and passive } \\
\text { robot-supported } \\
\text { gait }\end{array}$ & $\begin{array}{l}\text { No health } \\
\text { impairments; } \\
\text { Sample size: } \\
\text { 14; Exclusion: } \\
\text { 1; Male: 8; } \\
\text { Female: 7; Age: } \\
22 \text { to } 28 \\
\text { (average: } \\
24.3 \pm 2.7 \text { ) } \\
\text { right-handed }\end{array}$ & $\begin{array}{l}\mathrm{Mu}(8-12 \mathrm{~Hz}) \text { and } \\
\text { beta }(18-21 \mathrm{~Hz}) \\
\text { rhythms are } \\
\text { suppressed during } \\
\text { active walking } \\
\text { compared to } \\
\text { passive walking. }\end{array}$ & $\begin{array}{l}\text { Significant } \\
\text { differences in } \\
\text { cortical activation } \\
\text { between active and } \\
\text { passive robotic } \\
\text { walking support the } \\
\text { evaluation of brain } \\
\text { monitoring } \\
\text { techniques and } \\
\text { brain-computer } \\
\text { interface } \\
\text { technologies to } \\
\text { improve gait } \\
\text { restoration } \\
\text { therapies in a } \\
\text { top-down } \\
\text { approach. }\end{array}$ \\
\hline $\begin{array}{l}\text { Youssofzadeh } \\
\text { et al., } 2014\end{array}$ & $\begin{array}{l}\text { Directed neural } \\
\text { connectivity changes in } \\
\text { robot-assisted gait } \\
\text { training: A partial } \\
\text { Granger causality } \\
\text { analysis }\end{array}$ & $\begin{array}{l}\text { ALEX II on } \\
\text { non-dominant leg } \\
\text { (left) }\end{array}$ & $\begin{array}{l}\text { Speed: } 0.87 \pm 0.15 \\
\text { m/s. } 10 \text { training } \\
\text { paradigms Rest: } 2 \text { - } \\
4 \text { min. between } \\
\text { trials Haptic and } \\
\text { visual guidance: } \\
100 \% .\end{array}$ & $\begin{array}{l}\text { EEG; g.tec's; } \\
\text { g.USBamp; } 16 \\
\text { electrodes; } \\
\text { Electrode } \\
\text { placement: 10-20 } \\
\text { system }\end{array}$ & $\begin{array}{l}\text { PGC to elucidate } \\
\text { the functional } \\
\text { connectivity of EEG } \\
\text { signals in RAGT; } \\
\text { PSD for validity } \\
\text { check }\end{array}$ & $\begin{array}{l}\text { No health } \\
\text { impairments; } \\
\text { Sample size: } 6 \\
\text { male subjects } \\
\text { Age: } \\
26.5 \pm 6.5 \\
\text { years; Weight: } \\
77.8 \pm 9.7 \mathrm{~kg} ; \\
\text { Body height: } \\
1.79 \pm 0.04 \mathrm{~m}\end{array}$ & $\begin{array}{l}\text { The results showed } \\
\text { a strong causal } \\
\text { interaction between } \\
\text { the lateral motor } \\
\text { cortical areas. } \\
\text { A front-parietal } \\
\text { connection was } \\
\text { found in all robotic } \\
\text { training units. After } \\
\text { training a causal } \\
\text { "top-down" } \\
\text { cognitive control } \\
\text { was found. }\end{array}$ & $\begin{array}{l}\text { Causal "top-down" } \\
\text { cognition control } \\
\text { indicates plasticity } \\
\text { in connectivity in } \\
\text { the respective brain } \\
\text { regions. }\end{array}$ \\
\hline
\end{tabular}


TABLE 1 | Continued

\begin{tabular}{|c|c|c|c|c|c|c|c|c|}
\hline Author(s) & Title & Robotic system & Method & EEG/fNIRS device & Parameter & Sample & Outcome & Conclusion \\
\hline $\begin{array}{l}\text { Youssofzadeh } \\
\text { et al., } 2016\end{array}$ & $\begin{array}{l}\text { Directed Functional } \\
\text { Connectivity in } \\
\text { Fronto-Centroparietal } \\
\text { Circuit Correlates with } \\
\text { Motor Adaptation in } \\
\text { Gait Training }\end{array}$ & $\begin{array}{l}\text { ALEX II on } \\
\text { dominant leg (right) }\end{array}$ & $\begin{array}{l}\text { Conditions: } \\
\text { Standing upright, } \\
\text { walking } \\
\text { unsupported on a } \\
\text { treadmill, } \\
\text { robot-assisted } \\
\text { walking with and } \\
\text { without the task of } \\
\text { adjusting the } \\
\text { changed footpath } \\
\text { on a screen. } 10 \\
\text { training paradigms } \\
\text { Speed: } \\
0.87 \mathrm{~km} / \mathrm{h} \pm 0.15 \\
\mathrm{~m} / \mathrm{s}\end{array}$ & $\begin{array}{l}\text { EEG; g.tec's; } \\
\text { g.USBamp; } 16 \\
\text { electrodes; } \\
\text { Sampling rate: } \\
512 \mathrm{~Hz} \text {; Electrode } \\
\text { placement: 10-20 } \\
\text { system; Additional: } \\
\text { EMG }\end{array}$ & $\begin{array}{l}\text { PGC to elucidate } \\
\text { the functional } \\
\text { connectivity of EEG } \\
\text { signals in RAGT; } \\
\text { PSD for validity } \\
\text { check }\end{array}$ & $\begin{array}{l}\text { No health } \\
\text { impairments; } \\
\text { Sample Size: } 6 \\
\text { male subjects; } \\
\text { Age: } \\
26.5 \pm 6.5 \\
\text { years; Weight: } \\
77.8 \pm 9.7 \mathrm{~kg} ; \\
\text { Body height: } \\
1.79 \pm 0.04 \mathrm{~m}\end{array}$ & $\begin{array}{l}\text { PGC analysis } \\
\text { showed improved } \\
\text { connectivity near } \\
\text { sensorimotor areas } \\
\text { (C3, CP3) during } \\
\text { standing while } \\
\text { additional } \\
\text { connectivity near } \\
\text { central (CPz) and } \\
\text { frontal (Fz) areas } \\
\text { during walking } \\
\text { compared to } \\
\text { standing. } \\
\text { Significant fronto- } \\
\text { centroparietal } \\
\text { causal effects both } \\
\text { in training and after } \\
\text { training. Strong } \\
\text { correlations } \\
\text { between kinematic } \\
\text { errors and fronto- } \\
\text { centroparietal } \\
\text { connectivity during } \\
\text { and after training. } \\
\text { PSD analysis } \\
\text { showed increase in } \\
\alpha \text { rhythms during } \\
\text { standing, and theta } \\
\text { and } \gamma \text { during } \\
\text { walking. }\end{array}$ & $\begin{array}{l}\text { Fronto- } \\
\text { centroparietal } \\
\text { connectivity is a } \\
\text { potential } \\
\text { neuromarker for } \\
\text { motor learning and } \\
\text { adaptation in } \\
\text { RAGT. }\end{array}$ \\
\hline
\end{tabular}


TABLE 1 | Continued

\begin{tabular}{|c|c|c|c|c|c|c|c|c|}
\hline Author(s) & Title & Robotic system & Method & EEG/fNIRS device & Parameter & Sample & Outcome & Conclusion \\
\hline Kim et al., 2016 & $\begin{array}{l}\text { Best facilitated cortical } \\
\text { activation during } \\
\text { different stepping, } \\
\text { treadmill, and } \\
\text { robot-assisted walking } \\
\text { training paradigms and } \\
\text { speeds: A functional } \\
\text { near-infrared } \\
\text { spectroscopy } \\
\text { neuroimaging study }\end{array}$ & Lokomat & $\begin{array}{l}\text { Randomized, } \\
\text { based on a block } \\
\text { design; } 3 \\
\text { Conditions: } \\
\text { Conventional } \\
\text { walking, TW, RAGT; } \\
\text { Speed: (1) } \\
\text { self-selected, (2+3) } \\
\text { 1,5, 2.0, 2.5, } \\
\text { 3,0 km/h; GF: } \\
\text { 100\%. BWS: 50\% }\end{array}$ & fNIRS 31-channels & $\begin{array}{l}\text { Cerebral } \\
\text { hemodynamic } \\
\text { changes } \\
\text { associated with } \\
\text { cortical movement } \\
\text { network regions in } \\
\text { the primary SMC, } \\
\text { PMC, SMA, PFC } \\
\text { and SAC }\end{array}$ & $\begin{array}{l}\text { No health } \\
\text { impairments; } \\
\text { Sample size: } \\
\text { 14; Men: 8; } \\
\text { Women: 6; } \\
\text { Age: } \\
30.06 \pm 4.53 \\
\text { years } \\
\text { right-handers }\end{array}$ & $\begin{array}{l}\text { More global } \\
\text { activation of the } \\
\text { motion network } \\
\text { (SMC, PMC, SMA) } \\
\text { during RAGT } \\
\text { compared to } \\
\text { conventional and } \\
\text { treadmill walking. } \\
\text { Positive correlation } \\
\text { of speed and } \\
\text { activity of the } \\
\text { movement } \\
\text { network. }\end{array}$ & $\begin{array}{l}\text { RAGT provides the } \\
\text { best cortical } \\
\text { activation } \\
\text { associated with } \\
\text { motor control. }\end{array}$ \\
\hline $\begin{array}{l}\text { Simis et al., } \\
2018\end{array}$ & $\begin{array}{l}\text { Using Functional near } \\
\text { Infrared Spectroscopy } \\
\text { (fNIRS) to assess brain } \\
\text { activity of spinal cord } \\
\text { injury patient, during } \\
\text { robot-assisted gait }\end{array}$ & Lokomat & $\begin{array}{l}\text { Conditions: } \\
\text { Standing (resting } \\
\text { position) and } \\
\text { walking in the } \\
\text { Lokomat }\end{array}$ & $\begin{array}{l}\text { fNIRS } 32 \text { optodes: } \\
16 \text { emitters, } 16 \\
\text { detectors; } \\
\text { Placement: 10-20 } \\
\text { system }\end{array}$ & $\begin{array}{l}\text { Cerebral } \\
\text { hemodynamic } \\
\text { changes in the } \\
\text { motor cortex of } \\
\text { both hemispheres. } \\
\text { Relative change in } \\
\text { concentration of } \\
\text { oxy- and } \\
\text { deoxyhemoglobin }\end{array}$ & $\begin{array}{l}\text { Patients with } \\
\text { spinal cord } \\
\text { injury; Number } \\
\text { of test persons: } \\
3 \text { patients }\end{array}$ & $\begin{array}{l}\text { Two of the patients } \\
\text { had an increased } \\
\text { activation in M1 } \\
\text { during the RAGT, } \\
\text { compared to the } \\
\text { standing position. } \\
\text { One of the patients } \\
\text { showed no } \\
\text { changes in M1 } \\
\text { brain activity. }\end{array}$ & $\begin{array}{l}\text { fNIRS is suitable for } \\
\text { measuring the brain } \\
\text { activity of } \mathrm{SCl} \\
\text { patients during } \\
\text { robotic walking. } \\
\text { Results indicate an } \\
\text { increased } \\
\text { involvement of the } \\
\text { motor cortical areas } \\
\text { during walking. }\end{array}$ \\
\hline
\end{tabular}

ALEX, active leg exoskeleton; BWS, body weight support; CSE, cortical spinal excitability; DAR, delta-alpha EEG power ratio; EGT, ekso-gait training; ERD, event-related desynchronization; ERS, event-related synchronization; ERSPs, event-related spectral perturbation; FPEC, frontoparietal effective connectivity; GAR, gait assistance robot; GF, guidance force; HMI, human-motion-intention; M1, primary motor cortex; OGT, over ground gait training; PFC, prefrontal cortex; PGC, time-domain partial Granger causality; PMC, primary motor cortex; PSD, power spectral density; RAGT, robot-assisted gait training; SAC, sensory association area; SEMG, surface electromyography; SEP, sensory evoked potentials; SMA, supplementary motor area; SMC, sensorimotor cortex; SMI, sensorimotor integration; TBI, traumatic brain injury; TMS, transcranial magnetic stimulation. 
less guidance force compared to complete guidance (Knaepen et al., 2015). Furthermore, low gamma (24-40 Hz; Wagner et al., 2012; Seeber et al., 2014) and high gamma (70$90 \mathrm{~Hz}$ ) amplitudes were modulated depending on gait cycle phases, but conversely to each other (Seeber et al., 2015). Youssofzadeh et al. $(2014,2016)$ reported significant frontocentroparietal connectivity during and after RAGT proposing potential neuromarkers for motor learning and adaptation in RAGT. Other investigations aimed to eliminate EEG artifacts by using REMOV, a method that combines various approaches and established ICA based routines to remove contaminations, thus providing a procedure to use EEG as an imaging technique during RAGT (Artoni et al., 2012). Clinical studies were conducted either with patients suffering from stroke (Calabrò et al., 2018; Contreras-Vidal et al., 2018) or traumatic brain injury (Lapitskaya et al., 2011). In a prospective, pre-post, randomized RAGT based clinical study with 40 stroke patients, improved frontoparietal effective connectivity (FPEC) after eight weeks of Ekso ${ }^{\text {TM }}$ gait training compared to conventional training was observed (Calabrò et al., 2018). Contreras-Vidal et al. (2018) showed changes in neuroelectric cortical activity patterns during post-stroke rehabilitation, thus, demonstrating the feasibility of decoding walking from brain activity.

In the two fNIRS studies, hemodynamic changes in response to RAGT were investigated in healthy subjects (Kim et al., 2016) as well as in patients with spinal cord injury (Simis et al., 2018). Kim et al. (2016) compared cortical activation during different stepping, treadmill, and RAGT paradigms and speeds. Cerebral hemodynamic changes were determined in cortical locomotor network areas. Elevated global locomotor network activation was observed during RAGT compared to stepping or treadmill walking, thus leading to the conclusion that RAGT facilitated greater cortical activation associated with locomotor control than without RAGT (Kim et al., 2016). The feasibility of fNIRS in patients suffering from spinal cord injury were shown by enhanced activation in motor cortex during RAGT compared to standing. This supports the assumption of enhanced involvement of motor cortical areas during RAGT (Simis et al., 2018).

In summary, the majority of EEG based studies demonstrated suppressed mu and beta oscillations while in fNIRS studies increased Hboxy changes in sensorimotor areas during RAGT were observed. Nevertheless, it must be noted that the studies differ significantly in terms of their methodology such as robotic device, EEG/fNIRS system, design, subjects and parameters that lead to heterogeneous results (see Table 1). To date, the complexity of our brain still leads to a limited understanding of the relation among brain activation and behavior as well as dysfunction and disorder. While measuring different perspectives of brain activity, the utilization of fused EEG-fNIRS helps to identify more features correlated with brain activation and brain connectivity in the complex process of gait. However, questions remain how the signals are related, and which advantages the fusion provides in terms of studying brain dysfunction due to gait disorders as well as neurophysiological changes during robotic gait rehabilitation?

\section{FUSED EEG-fNIRS TO MONITOR BRAIN ACTIVITY}

Both EEG and fNIRS are comparatively inexpensive and portable, they have unique, complementary advantages without interfering with each other, thus leading to an increased interest for fused EEG-fNIRS in rehabilitation research. The connection of the systems can be ensured either via wired trigger signals, which are split into various synchronized signals, or via connected networks. For the latter, the signals from different devices could be synchronized using systems (e.g., Lab Streaming Layer) where the networking and time-synchronization of the signals are handled to offer a uniform recording of measurement time series (Chadwick, 2018). Different approaches have been developed by taking advantage of simultaneous multimodal measurement.

First, fused EEG-fNIRS can be used for the investigation of how neuronal changes are related to neurovascular coupling. During a motor task, increased Hboxy concentration was accompanied by a decrease in Hbdeoxy and a decrease in alpha and beta power (Lachert et al., 2017). Koch et al. (2008) also reported a correlation between high individual alpha frequency (IAF) and low Hboxy response (Koch et al., 2008). Furthermore, Berger et al. (2018) extended the relation by demonstrating an increase of EEG alpha power following 10 and $20 \mathrm{~Hz}$ transcranial alternating current stimulation (tACS), which was accompanied by a decrease in Hboxy (Berger et al., 2018). Based on previous studies investigating neural correlates of walking or RAGT, it can be expected that suppressed alpha and beta oscillations in the motor cortical network are accompanied by increases in Hboxy. However, numerous questions regarding the correlation of neuronal activities and vascular changes (e.g., co-localization / time lag of the correlated changes or consistency of changes in connectivity; Functional Brain Imaging with EEG and fNIRS, o.d.) remain.

Second, fused EEG-fNIRS provides detailed spatio-temporal information on neurophysiological activity through different strengths and weaknesses of EEG and fNIRS, thus leading in improved distinctions of conditions such as rest and task (Leamy et al., 2011; Fazli et al., 2012; Khan et al., 2018). Leamy et al. (2011) investigated hemodynamic and electrical responses during motor imagery (MI) and rest periods. Classification of 2-dimensional EEG feature spaces (change in mu and beta power from rest to $\mathrm{MI}$ ) and fNIRS feature spaces (change in average amplitude of Hboxy and Hbdeoxy from rest to $\mathrm{MI}$ ) was performed using linear discriminant analysis (LDA), at first separately and in combination afterward. By employing a supplemental measurement modality through combining EEG-fNIRS, more information about the underlying neurovascular relationship can be achieved (Leamy et al., 2011). If the classification of fNIRS features can complement EEG classifications were also examined in a real-time sensor motor-rhythm based brain-computer interface (BCI) paradigm with executed movements and MI. By using LDA, fused EEG-fNIRS provide complementary spatiotemporal information with a significantly improved classification 
accuracy (Fazli et al., 2012). Furthermore, it would be possible to use data by one technique to reduce artifacts of the other due to the independence between electrical and optical measurements but up to date, these possibilities are not yet sufficiently proven. By the fact that fNIRS is limited due to the long-term delay of the hemodynamic response (Fazli et al., 2012) and requires a minimum distance between sources and detectors that detectors are not suffering from "source blinding", improving fNIRS through supplementing EEG data might be more useful than adding fNIRS to EEG (Leamy et al., 2011).

In summary, the independence between electrical and optical measurements can be used advantageously to (1) obtain detailed spatio-temporal information, (2) improve classification accuracy and (3) reduce effects of movements artifacts with the aim to improve the reliability and robustness of signal interpretation (Shin et al., 2018). Nevertheless, the relation of EEG and fNIRS is not yet fully understood (Leamy et al., 2011) and challenges of the combined EEG-fNIRS approach arise. For instance, electrical and hemodynamic signals are not necessarily coupled. On one side, physiological process (e.g., neurotransmitter synthesis) can cause hemodynamic changes without electrophysiological activity. On the other side, changes in metabolic activity may not be detectable if EEG activity is transient (Yuan et al., 2014). Furthermore, EEG and fNIRS measurements make great demands on study designs due to the susceptibility to motion and light interference (Vitorio et al., 2017) which is of high relevance especially for clinical studies. In addition, there is no uniform, standardized procedure due to many different methods regarding signal processing and analysis which makes the comparability and interpretation difficult. According to this, the combination of EEG and fNIRS is very complex and still in its infancy despite the technical progress.

However, based on the knowledge that multi-modal measurement has the capacity to analyze neurovascular coupling more accurately, fused EEG-fNIRS might be beneficial to basic neuroscientific research as well as clinical applications (Muthalib et al., 2013).

\section{FUTURE PROSPECTS FOR FUSED EEG-fNIRS IN RAGT}

Regarding RAGT, fused EEG-fNIRS provides a detailed insight into how locomotor control and gait recovery is characterized by brain signals. On one side, the understanding of the underlying mechanisms of RAGT might be a further step toward scientific-based evidence for improved gait recovery. Based on brain signals, optimal training parameters and settings (e.g., BWS, GF; Knaepen et al., 2015) as well as augmented feedback (Wagner et al., 2014; Calabrò et al., 2017) can be determined for individualized gait therapy protocols. Wagner et al. (2014) examined the impact of visual feedback on EEG pattern during RAGT and presented that movement related interactive feedback in virtual environment (VE) significantly increase brain activity in premotor and parietal areas due to motor planning and visuomotor processes compared to movement unrelated feedback (Wagner et al., 2014). Thus, robotassisted gait rehabilitation can be further improved, for example by selecting specific feedback based on the underlying network activity in order to promote individuals voluntary drive which is crucial for motor learning.

On other side, EEG-fNIRS signals can be used to expand and integrate further approaches such as EEG/fNIRS controlled gait therapy in brain machine interface (BMI) paradigms or noninvasive brain stimulation (NIBS) during RAGT that are guided by oscillatory or hemodynamic activity (see Figure 2).

In BMI, cortical activity associated with movements were identified and used to directly control external devices (GarcíaCossio et al., 2015; Donati et al., 2016; Teo et al., 2016). Donati et al. (2016) investigated the effects of long-term training with a BMI-based gait protocol on motor recovery and restoring mobility in paraplegic patients. Eight chronic paralyzed patients performed a BMI neurorehabilitation paradigm including virtual reality training, enriched visualtactile feedback, and walking with EEG-controlled robotic devices. After 12 months of training, paraplegic patients improved their somatic sensation and walking abilities. It was hypothesized that this unprecedented neurological recovery resulted from both cortical and spinal cord plasticity triggered by long-term training with BMI (Donati et al., 2016). For the future of non-invasive, portable, and wearable BMIs, researchers suggest the use of hybrid EEG-fNIRS systems, as they have been shown to be superior to the use of EEG-BCIs and fNIRS-BCIs alone (Fazli et al., 2012; Khan et al., 2014; Koo et al., 2015; Naseer and Hong, 2015).

Another future prospect could include the feedback of cortical activation pattern measured by fused EEG-fNIRS that is used to identify regions of hypo- or hyperactivity to guide NIBS protocols (Teo et al., 2016; Berger et al., 2018). Transcranial electrical stimulation (tES) is regarded as one of the most well-known forms of NIBS who's primarily researched modalities are transcranial direct current stimulation (tDCS) and transcranial alternating current stimulation (tACS; Paulus, 2011; Yavari et al., 2018). By applying weak current through the scalp, tES represent promising tools for the induction of acute or long-lasting effects on excitability or brain network dynamics, thus investigating the causal relationship between brain activity, motor functions (Yavari et al., 2018) and potentially enhancing motor learning processes (Reis and Fritsch, 2011; Sugata et al., 2018). tES can be set up as portable and wireless systems, thus having complimentary capabilities as well as EEG and fNIRS (McKendrick et al., 2015). In this way, combining tES with fused EEG-fNIRS is suitable for modulating pathological brain pattern to enhance neuroplasticity during RAGT, thereby representing an avenue for clinical applications (McKendrick et al., 2015). For example, fused EEG/fNIRS allow the identification of hypo- or hyperactivity accompanied by gait disorders that might help to determine and guide brain stimulation protocols. Thus, tES may be applied during RAGT to modulate neuronal networks supporting gait rehabilitation (Teo et al., 2016). Closed-loop tES during RAGT as well as BMI guided by fused EEG-fNIRS represent 


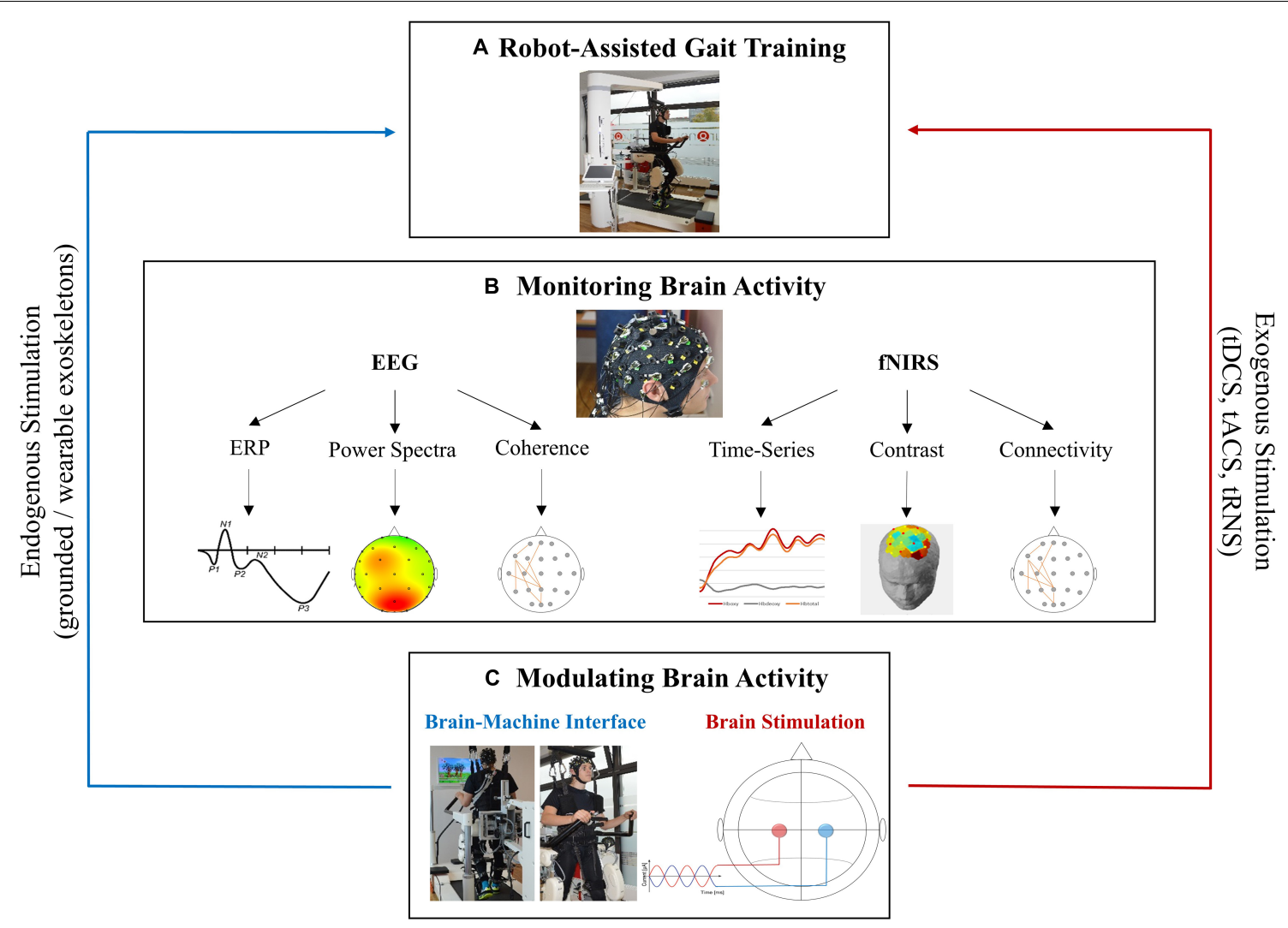

FIGURE 2 | Future perspectives for fused EEG-fNIRS in robotic gait rehabilitation. (A) Robotic device Lokomat (Hocoma, Switzerland) for RAGT. (B) EEG and fNIRS provide the monitoring of neurophysiological processes occurring during RAGT by measuring different perspectives of brain activity. Electrophysiological (EEG) and haemodynamic (fNIRS) signals can be processed in various ways. Data from each electrode/channel can be extracted over time (e.g., ERPs/time-series), as an average over time (e.g., power spectra/contrasts) or they can be correlated to represent functional connectivity (e.g., coherence/connectivity). (C) Brain activity measured by EEG or fNIRS serves as a basis for brain modulation such as BCl/BMI which may be a form of endogenous brain stimulation as well as for brain stimulation techniques were a weak current is applied through the skull to modulate brain activity. Subjects appearing in the figures provided informed written consent to the publication of identifying images in an online open access publication.

two approaches for the implementation of individualized neurorehabilitation which is essential for success in motor recovery (McKendrick et al., 2015).

\section{CONCLUSION}

EEG and fNIRS measure different perspectives of brain activity. Therefore, fused EEG-fNIRS provide detailed spatiotemporal information correlated with brain activity and connectivity. Based on the reviewed studies investigating brain activity following RAGT as well as studies using multimodal approaches, we conclude that fused EEG-fNIRS has the potential to characterize the complex neurovascular coupling mechanisms associated with gait disorders due to neurological diseases as well as robotic rehabilitation in more detail than by using one modality alone. Thus, neuroplastic changes due to RAGT that were measured by fused EEG-fNIRS might contribute to scientifically prove the effectiveness of RAGT. Furthermore, revealing brain activity underlying RAGT is essential for the adjustment of therapy protocols and the guidance of further interventions such as BMI or NIBS. Fused EEG-fNIRS approaches the goal of individualized, closed-loop RAGT that optimizes the outcome and efficacy of gait recovery, thereby fostering independent living and improved quality of life for neurological patients. Despite technical advances (e.g., signal processing, data synchronization), fused EEG-fNIRS remains complex and poses challenges that require further innovations to determine the potential during RAGT in clinical and in non-clinical environment.

\section{AUTHOR CONTRIBUTIONS}

$\mathrm{AB}$ primarily contributed to the conceptualization of the review, performed the literature research, interpreted the data, wrote the manuscript, approved the final version of the manuscript, and acted as corresponding author. SM contributed to the literature research and wrote a part of the manuscript. FH, FS, and MD substantially contributed to the data interpretation and critically revised the manuscript. 


\section{REFERENCES}

Artoni, F., Chisari, C., Menicucci, D., Fanciullacci, C., and Micera, S. (2012). "REMOV: EEG artifacts removal methods during Lokomat lower-limb rehabilitation," in Proceedings of the 4th IEEE RAS \& EMBS International Conference on Biomedical Robotics and Biomechatronics (BioRob), (Piscataway, NJ: IEEE), 992-997.

Beer, S., Aschbacher, B., Manoglou, D., Gamper, E., Kool, J., and Kesselring, J. (2008). Robot-assisted gait training in multiple sclerosis: a pilot randomized trial. Mult. Scler. 14, 231-236. doi: 10.1177/1352458507082358

Benito-Penalva, J., Edwards, D. J., Opisso, E., Cortes, M., Lopez-Blazquez, R., Murillo, N., et al. (2012). Gait training in human spinal cord injury using electromechanical systems: effect of device type and patient characteristics. Arch. Phys. Med. Rehabil. 93, 404-412. doi: 10.1016/j.apmr.2011.08.028

Berger, A., Pixa, N. H., Steinberg, F., and Doppelmayr, M. (2018). Brain oscillatory and hemodynamic activity in a bimanual coordination task following transcranial alternating current stimulation (tACS): a combined EEGfNIRS study. Front. Behav. Neurosci. 12:67. doi: 10.3389/fnbeh.2018.00067

Brigadoi, S., Ceccherini, L., Cutini, S., Scarpa, F., Scatturin, P., Selb, J., et al. (2014). Motion artifacts in functional near-infrared spectroscopy: a comparison of motion correction techniques applied to real cognitive data. Neuroimage 85(Pt 1), 181-191. doi: 10.1016/j.neuroimage.2013.04.082

Bulea, T. C., Kim, J., Damiano, D. L., Stanley, C. J., and Park, H.-S. (2015). Prefrontal, posterior parietal and sensorimotor network activity underlying speed control during walking. Front. Hum. Neurosci. 9:247. doi: 10.3389/fnhum. 2015.00247

Calabrò, R. S., Cacciola, A., Bertè, F., Manuli, A., Leo, A., Bramanti, A., et al. (2016). Robotic gait rehabilitation and substitution devices in neurological disorders: where are we now? Neurol. Sci. 37, 503-514. doi: 10.1007/s10072-016-2474-4

Calabrò, R. S., Naro, A., Russo, M., Bramanti, P., Carioti, L., Balletta, T., et al. (2018). Shaping neuroplasticity by using powered exoskeletons in patients with stroke: a randomized clinical trial. J. Neuroeng. Rehabil. 15:35. doi: 10.1186/ s12984-018-0377-8

Calabrò, R. S., Naro, A., Russo, M., Leo, A., Luca, R., de Balletta, T., et al. (2017). The role of virtual reality in improving motor performance as revealed by EEG: a randomized clinical trial. J. Neuroeng. Rehabil. 14:53. doi: 10.1186/s12984017-0268-4

Chadwick, B. (2018). Lab Streaming Layer. Available at: https://github.com/sccn/ labstreaminglayer/wiki (accessed November 26, 2018).

Chen, L.-C., Sandmann, P., Thorne, J. D., Herrmann, C. S., and Debener, S. (2015). Association of concurrent fNIRS and EEG signatures in response to auditory and visual stimuli. Brain Topogr. 28, 710-725. doi: 10.1007/s10548-015-0 424-8

Contreras-Vidal, J. L., Bortole, M., Zhu, F., Nathan, K., Venkatakrishnan, A., Francisco, G. E., et al. (2018). Neural decoding of robot-assisted gait during rehabilitation after stroke. Am. J. Phys. Med. Rehabil. 97, 541-550. doi: 10.1097/ PHM.0000000000000914

Donati, A. R. C., Shokur, S., Morya, E., Campos, D. S. F., Moioli, R. C., Gitti, C. M., et al. (2016). Long-term training with a brain-machine interface-based gait protocol induces partial neurological recovery in paraplegic patients. Sci. Rep. 6:30383. doi: 10.1038/srep30383

Esquenazi, A., Lee, S., Packel, A. T., and Braitman, L. (2013). A randomized comparative study of manually assisted versus robotic-assisted body weight supported treadmill training in persons with a traumatic brain injury. J. Inj. Funct. Rehabil. 5, 280-290. doi: 10.1016/j.pmrj.2012.10.009

Fazli, S., Mehnert, J., Steinbrink, J., Curio, G., Villringer, A., Müller, K.-R., et al. (2012). Enhanced performance by a hybrid NIRS-EEG brain computer interface. Neuroimage 59, 519-529. doi: 10.1016/j.neuroimage.2011.07.084

Ferrari, M., Mottola, L., and Quaresima, V. (2004). Principles, techniques, and limitations of near infrared spectroscopy. Can. J. Appl. Physiol. 29, 463-487. doi: 10.1139/h04-031

Functional Brain Imaging with EEG and fNIRS (o.d.). Available at https://www. cmu.edu/bme/biophotonics/research/eeg-fnirs.html

García-Cossio, E., Severens, M., Nienhuis, B., Duysens, J., Desain, P., Keijsers, N., et al. (2015). Decoding sensorimotor rhythms during robotic-assisted treadmill walking for brain computer interface (BCI) applications. PLoS One 10:e0137910. doi: 10.1371/journal.pone.0137910
Gramann, K., Gwin, J. T., Bigdely-Shamlo, N., Ferris, D. P., and Makeig, S. (2010). Visual evoked responses during standing and walking. Front. Hum. Neurosci. 4:202. doi: 10.3389/fnhum.2010.00202

Gramann, K., Gwin, J. T., Ferris, D. P., Oie, K., Jung, T.-P., Lin, C.-T., et al. (2011). Cognition in action: imaging brain/body dynamics in mobile humans. Rev. Neurosci. 22, 593-608. doi: 10.1515/RNS.2011.047

Gwin, J. T., Gramann, K., Makeig, S., and Ferris, D. P. (2010). Removal of movement artifact from high-density EEG recorded during walking and running. J. Neurophysiol. 103, 3526-3534. doi: 10.1152/jn.00105.2010

Gwin, J. T., Gramann, K., Makeig, S., and Ferris, D. P. (2011). Electrocortical activity is coupled to gait cycle phase during treadmill walking. Neuroimage 54, 1289-1296. doi: 10.1016/j.neuroimage.2010.08.066

Hamacher, D., Herold, F., Wiegel, P., Hamacher, D., and Schega, L. (2015). Brain activity during walking: a systematic review. Neurosci. Biobehav. Rev. 57, 310-327. doi: 10.1016/j.neubiorev.2015.08.002

Harada, T., Miyai, I., Suzuki, M., and Kubota, K. (2009). Gait capacity affects cortical activation patterns related to speed control in the elderly. Exp. Brain Res. 193, 445-454. doi: 10.1007/s00221-008-1643-y

Husemann, B., Müller, F., Krewer, C., Heller, S., and Koenig, E. (2007). Effects of locomotion training with assistance of a robot-driven gait orthosis in hemiparetic patients after stroke: a randomized controlled pilot study. Stroke 38, 349-354. doi: 10.1161/01.STR.0000254607.48765.cb

Kays, J. L., Hurley, R. A., and Taber, K. H. (2012). The dynamic brain: neuroplasticity and mental health. J. Neuropsych. Clin. Neurosci. 24, 118-124. doi: 10.1176/appi.neuropsych.24.1.118

Khan, M. J., Ghafoor, U., and Hong, K.-S. (2018). Early detection of hemodynamic responses using EEG: a hybrid EEG-fNIRS study. Front. Hum. Neurosci. 12:479. doi: 10.3389/fnhum.2018.00479

Khan, M. J., Hong, M. J., and Hong, K.-S. (2014). Decoding of four movement directions using hybrid NIRS-EEG brain-computer interface. Front. Hum. Neurosci. 8:244. doi: 10.3389/fnhum.2014.00244

Kim, H. Y., Yang, S. P., Park, G. L., Kim, E. J., and You, J. S. (2016). Best facilitated cortical activation during different stepping, treadmill, and robotassisted walking training paradigms and speeds: a functional near-infrared spectroscopy neuroimaging study. Neurorehabilitation 38, 171-178. doi: 10. 3233/NRE- 161307

Kline, J. E., Huang, H. J., Snyder, K. L., and Ferris, D. P. (2015). Isolating gaitrelated movement artifacts in electroencephalography during human walking. J. Neural Eng. 12:46022. doi: 10.1088/1741-2560/12/4/046022

Knaepen, K., Mierau, A., Swinnen, E., Fernandez Tellez, H., Michielsen, M., Kerckhofs, E., et al. (2015). Human-robot interaction: does robotic guidance force affect gait-related brain dynamics during robot-assisted treadmill walking? PLoS One 10:e0140626. doi: 10.1371/journal.pone.0140626

Koch, S. P., Koendgen, S., Bourayou, R., Steinbrink, J., and Obrig, H. (2008). Individual alpha-frequency correlates with amplitude of visual evoked potential and hemodynamic response. Neuroimage 41, 233-242. doi: 10.1016/j. neuroimage.2008.02.018

Koo, B., Lee, H.-G., Nam, Y., Kang, H., Koh, C. S., Shin, H.-C., et al. (2015). A hybrid NIRS-EEG system for self-paced brain computer interface with online motor imagery. J. Neurosci. Methods 244, 26-32. doi: 10.1016/j.jneumeth.2014. 04.016

Lachert, P., Janusek, D., Pulawski, P., Liebert, A., Milej, D., and Blinowska, K. J. (2017). Coupling of Oxy- and deoxyhemoglobin concentrations with EEG rhythms during motor task. Sci. Rep. 7:15414. doi: 10.1038/s41598-017-157702

Lapitskaya, N., Nielsen, J. F., and Fuglsang-Frederiksen, A. (2011). Robotic gait training in patients with impaired consciousness due to severe traumatic brain injury. Brain Inj. 25, 1070-1079. doi: 10.3109/02699052.2011.607782

Leamy, D. J., Collins, R., and Ward, T. E. (2011). "Combining fNIRS and EEG to Improve Motor Cortex Activity Classification during an Imagined MovementBased Task," in Proceedings of the Foundations of Augmented Cognition: Directing the Future of Adaptive Systems; 6th International Conference, FAC 2011, Held as Part of HCI International 2011, eds D. D. Schmorrow and C. M. Fidopiastis (Berlin: Springer), 177-185. doi: 10.1007/978-3-642-21 852-1_22

Makeig, S., Debener, S., Onton, J., and Delorme, A. (2004). Mining event-related brain dynamics. Trends Cogn. Sci. 8, 204-210. doi: 10.1016/j.tics.2004.03.008 
Makeig, S., Gramann, K., Jung, T.-P., Sejnowski, T. J., and Poizner, H. (2009). Linking brain, mind and behavior. Int. J. Psychophysiol. 73, 95-100. doi: 10. 1016/j.ijpsycho.2008.11.008

McKendrick, R., Parasuraman, R., and Ayaz, H. (2015). Wearable functional near infrared spectroscopy (fNIRS) and transcranial direct current stimulation (tDCS): expanding vistas for neurocognitive augmentation. Front. Syst. Neurosci. 9:27. doi: 10.3389/fnsys.2015.00027

Merzagora, A. C., Izzetoglu, M., Polikar, R., Weisser, V., Onaral, B., and Schultheis, M. T. (2009). "Functional Near-Infrared Spectroscopy and Electroencephalography: A Multimodal Imaging Approach," in Proceedings of the Foundations of Augmented Cognition: Neuroergonomics and Operational Neuroscience: 5th International Conference, FAC 2009, Held as Part of HCI International 2009, eds D. D. Schmorrow, V. Estabrooke, and M. Grootjen (Berlin: Springer), 417-426. doi: 10.1007/978-3-642-02812-0_50

Miyai, I., Tanabe, H. C., Sase, I., Eda, H., Oda, I., Konishi, I., et al. (2001). Cortical mapping of gait in humans: a near-infrared spectroscopic topography study. Neuroimage 14, 1186-1192. doi: 10.1006/nimg.2001.0905

Moher, D., Liberati, A., Tetzlaff, J., and Altman, D. G. (2009). Preferred reporting items for systematic reviews and meta-analyses: the PRISMA statement. PLoS Med. 6:e1000097. doi: 10.1371/journal.pmed.1000097

Muthalib, M., Anwar, A. R., Perrey, S., Dat, M., Galka, A., Wolff, S., et al. (2013). Multimodal integration of fNIRS, fMRI and EEG neuroimaging. Clin. Neurophysiol. 124, 2060-2062. doi: 10.1016/j.clinph.2013. 03.018

Nakanishi, Y., Wada, F., Saeki, S., and Hachisuka, K. (2014). Rapid changes in arousal states of healthy volunteers during robot-assisted gait training: a quantitative time-series electroencephalography study. J. Neuroeng. Rehabil. 11:59. doi: 10.1186/1743-0003-11-59

Naseer, N., and Hong, K.-S. (2015). fNIRS-based brain-computer interfaces: a review. Front. Hum. Neurosci. 9:3. doi: 10.3389/fnhum.2015.00003

Onton, J., Westerfield, M., Townsend, J., and Makeig, S. (2006). Imaging human EEG dynamics using independent component analysis. Neurosci. Biobehav. Rev. 30, 808-822. doi: 10.1016/j.neubiorev.2006.06.007

Pascual-Leone, A., Amedi, A., Fregni, F., and Merabet, L. B. (2005). The plastic human brain cortex. Ann. Rev. Neurosci. 28, 377-401. doi: 10.1146/annurev. neuro.27.070203.144216

Pascual-Leone, A., Freitas, C., Oberman, L., Horvath, J. C., Halko, M., Eldaief, M., et al. (2011). Characterizing brain cortical plasticity and network dynamics across the age-span in health and disease with TMS-EEG and TMS-fMRI. Brain Topogr. 24, 302-315. doi: 10.1007/s10548-011-0196-8

Paulus, W. (2011). Transcranial electrical stimulation (tES - tDCS; tRNS, tACS) methods. Neuropsychol. Rehabil. 21, 602-617. doi: 10.1080/09602011.2011. 557292

Petersen, T. H., Willerslev-Olsen, M., Conway, B. A., and Nielsen, J. B. (2012). The motor cortex drives the muscles during walking in human subjects. J. Physiol. 590, 2443-2452. doi: 10.1113/jphysiol.2012.227397

Pinti, P., Tachtsidis, I., Hamilton, A., Hirsch, J., Aichelburg, C., Gilbert, S., et al. (2018). The present and future use of functional near-infrared spectroscopy (fNIRS) for cognitive neuroscience. Ann. N.Y. Acad. Sci. doi: 10.1111/nyas. 13948 [Epub ahead of print].

Reis, J., and Fritsch, B. (2011). Modulation of motor performance and motor learning by transcranial direct current stimulation. Curr. Opin. Neurol. 24, 590-596. doi: 10.1097/WCO.0b013e32834c3db0

Robinson, A. K., Venkatesh, P., Boring, M. J., Tarr, M. J., Grover, P., and Behrmann, M. (2017). Very high density EEG elucidates spatiotemporal aspects of early visual processing. Sci. Rep. 7:16248. doi: 10.1038/s41598-01716377-3

Sanctis, P., de Butler, J. S., Malcolm, B. R., and Foxe, J. J. (2014). Recalibration of inhibitory control systems during walking-related dual-task interference: a mobile brain-body imaging (MOBI) study. Neuroimage 94, 55-64. doi: 10.1016/ j.neuroimage.2014.03.016

Sato, T., Nambu, I., Takeda, K., Aihara, T., Yamashita, O., Isogaya, Y., et al. (2016). Reduction of global interference of scalp-hemodynamics in functional nearinfrared spectroscopy using short distance probes. Neuroimage 141, 120-132. doi: 10.1016/j.neuroimage.2016.06.054

Seeber, M., Scherer, R., Wagner, J., and Müller-Putz, G. R. (2013). Spatial-spectral identification Of $\mathrm{M}$ And $\mathrm{B}$ eeg rhythm sourcrs during robot-assisted walking. Biomed. Eng. 58(Suppl.):1. doi: 10.1515/bmt-2013-4433
Seeber, M., Scherer, R., Wagner, J., Solis-Escalante, T., and Müller-Putz, G. R. (2014). EEG beta suppression and low gamma modulation are different elements of human upright walking. Front. Hum. Neurosci. 8:485. doi: 10.3389/ fnhum.2014.00485

Seeber, M., Scherer, R., Wagner, J., Solis-Escalante, T., and Müller-Putz, G. R. (2015). High and low gamma EEG oscillations in central sensorimotor areas are conversely modulated during the human gait cycle. Neuroimage 112, 318-326. doi: 10.1016/j.neuroimage.2015.03.045

Severens, M., Nienhuis, B., Desain, P., and Duysens, J. (2012). Feasibility of measuring event related desynchronization with electroencephalography during walking. Conf. Proc. IEEE Eng. Med. Biol. Soc. 2012, 2764-2767. doi: 10.1109/EMBC.2012.6346537

Shin, J., Kwon, J., Choi, J., and Im, C.-H. (2017). Performance enhancement of a brain-computer interface using high-density multi-distance NIRS. Sci. Rep. 7:16545. doi: 10.1038/s41598-017-16639-0

Shin, J., Lühmann, A., von Kim, D.-W., Mehnert, J., Hwang, H.-J., and Müller, K.-R. (2018). Simultaneous acquisition of EEG and NIRS during cognitive tasks for an open access dataset. Sci. Data 5:180003. doi: 10.1038/sdata.2 018.3

Simis, M., Santos, K., Sato, J., Fregni, F., and Battistella, L. (2018). T107. using functional near infrared spectroscopy (fNIRS) to assess brain activity of spinal cord injury patient, during robot-assisted gait. Clin. Neurophysiol. 129, e43-e44. doi: 10.1016/j.clinph.2018.04.108

Snyder, K. L., Kline, J. E., Huang, H. J., and Ferris, D. P. (2015). Independent component analysis of gait-related movement artifact recorded using EEG electrodes during treadmill walking. Front. Hum. Neurosci. 9:639. doi: 10.3389/ fnhum.2015.00639

Solis Escalante, T., Wagner, J., Scherer, R., Grieshofer, P., and Müller-Putz, G. (2012). "Assessing participation during robotic assisted gait training based on EEG: feasibility study," in Poster Session Presented at 3rd TOBI Workshop Bringing BCIs to End-Users: Facing the Challenge - Evaluation, User Perspective, User Needs and Ethical Questions, Wuerzburg.

Stuart, S., Vitorio, R., Morris, R., Martini, D. N., Fino, P. C., and Mancini, M. (2018). Cortical activity during walking and balance tasks in older adults and in people with Parkinson's disease: a structured review. Maturitas 113, 53-72. doi: 10.1016/j.maturitas.2018.04.011

Stump, E. (2007). WHO report: millions have neurological disorders worldwide. Neurol. Today 7:25. doi: 10.1097/01.NT.0000269134.28964.7e

Sugata, H., Yagi, K., Yazawa, S., Nagase, Y., Tsuruta, K., Ikeda, T., et al. (2018). Modulation of motor learning capacity by transcranial alternating current stimulation. Neuroscience 391, 131-139. doi: 10.1016/j.neuroscience.2018.0 9.013

Swinnen, E., Baeyens, J.-P., Knaepen, K., Michielsen, M., Hens, G., Clijsen, R., et al. (2015). Walking with robot assistance: the influence of body weight support on the trunk and pelvis kinematics. disability and rehabilitation. Assist. Technol. 10, 252-257. doi: 10.3109/17483107.2014.888487

Swinnen, E., Duerinck, S., Baeyens, J.-P., Meeusen, R., and Kerckhofs, E. (2010). Effectiveness of robot-assisted gait training in persons with spinal cord injury: a systematic review. J. Rehabil. Med. 42, 520-526. doi: 10.2340/16501977-0538

Teo, W.-P., Muthalib, M., Yamin, S., Hendy, A. M., Bramstedt, K., Kotsopoulos, E., et al. (2016). Does a combination of virtual reality, neuromodulation and neuroimaging provide a comprehensive platform for neurorehabilitation? - a narrative review of the literature. Front. Hum. Neurosci. 10:284. doi: 10.3389/ fnhum.2016.00284

Turner, D. L., Ramos-Murguialday, A., Birbaumer, N., Hoffmann, U., and Luft, A. (2013). Neurophysiology of robot-mediated training and therapy: a perspective for future use in clinical populations. Front. Neurol. 4:184. doi: 10.3389/fneur. 2013.00184

Uçar, D. E., Paker, N., and Buðdaycı, D. (2014). Lokomat: a therapeutic chance for patients with chronic hemiplegia. Neurorehabilitation 34, 447-453. doi: 10.3233/NRE- 141054

Villa-Parra, A. C., Delisle-Rodríguez, D., López-Delis, A., Bastos-Filho, T., Sagaró, R., and Frizera-Neto, A. (2015). Towards a robotic knee exoskeleton control based on human motion intention through EEG and sEMGsignals. Proc. Manufact. 3, 1379-1386. doi: 10.1016/j.promfg.2015.07.296

Vitorio, R., Stuart, S., Rochester, L., Alcock, L., and Pantall, A. (2017). fNIRS response during walking - artefact or cortical activity? A systematic review. Neurosci. Biobehav. Rev. 83, 160-172. doi: 10.1016/j.neubiorev.2017.10.002 
Wagner, J., Makeig, S., Gola, M., Neuper, C., and Müller-Putz, G. (2016). Distinct $\beta$ band oscillatory networks subserving motor and cognitive control during gait adaptation. J. Neurosci. 36, 2212-2226. doi: 10.1523/JNEUROSCI.3543-15. 2016

Wagner, J., Solis-Escalante, T., Grieshofer, P., Neuper, C., Müller-Putz, G., and Scherer, R. (2012). Level of participation in robotic-assisted treadmill walking modulates midline sensorimotor EEG rhythms in able-bodied subjects. Neuroimage 63, 1203-1211. doi: 10.1016/j.neuroimage.2012.08.019

Wagner, J., Solis-Escalante, T., Scherer, R., Neuper, C., and Müller-Putz, G. (2014). It's how you get there: Walking down a virtual alley activates premotor and parietal areas. Front. Hum. Neurosci. 8:93. doi: 10.3389/fnhum.2014.00093

Yavari, F., Jamil, A., Mosayebi Samani, M., Vidor, L. P., and Nitsche, M. A. (2018). Basic and functional effects of transcranial electrical stimulation (tES)An introduction. Neurosci. Biobehav. Rev. 85, 81-92. doi: 10.1016/j.neubiorev. 2017.06.015

Youssofzadeh, V., Zanotto, D., Stegall, P., Naeem, M., Wong-Lin, K., Agrawal, S. K., et al. (2014). Directed neural connectivity changes in robot-assisted gait training: a partial Granger causality analysis. Conf. Proc. IEEE Eng. Med. Biol. Soc. 2014, 6361-6364. doi: 10.1109/EMBC.2014.6945083
Youssofzadeh, V., Zanotto, D., Wong-Lin, K., Agrawal, S. K., and Prasad, G. (2016). Directed functional connectivity in fronto-centroparietal circuit correlates with motor adaptation in gait training. IEEE Trans. Neural Syst. Rehabil. Eng. 24, 1265-1275. doi: 10.1109/TNSRE.2016.2551642

Yuan, Z., Zhang, J., and Lin, X. (2014). Technological Advances and Prospects in Multimodal Neuroimaging: Fusions of fNIRS-fMRI, fNIRS-EEG and fMRI-EEG. Saarbrücken: LAP LAMBERT Academic Publishing.

Conflict of Interest Statement: The authors declare that the research was conducted in the absence of any commercial or financial relationships that could be construed as a potential conflict of interest.

Copyright (C) 2019 Berger, Horst, Müller, Steinberg and Doppelmayr. This is an openaccess article distributed under the terms of the Creative Commons Attribution License (CC BY). The use, distribution or reproduction in other forums is permitted, provided the original author(s) and the copyright owner(s) are credited and that the original publication in this journal is cited, in accordance with accepted academic practice. No use, distribution or reproduction is permitted which does not comply with these terms. 